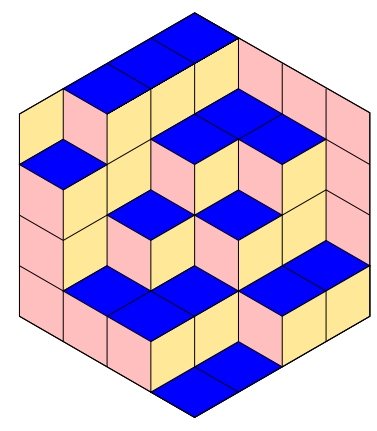

ALGEBRAIC COMBINATORICS

\title{
Bob Lutz
}

\section{Higher discrete homotopy groups of graphs}

Volume 4, issue 1 (2021), p. 69-88.

<http://alco.centre-mersenne.org/item/ALCO_2021__4_1_69_0>

(C) The journal and the authors, 2021.

Some rights reserved.

(c) BY This article is licensed under the

Creative Commons ATtribution 4.0 International LiCEnSE.

http://creativecommons.org/licenses/by/4.0/

Access to articles published by the journal Algebraic Combinatorics on the website http://alco.centre-mersenne.org/ implies agreement with the Terms of Use (http://alco.centre-mersenne.org/legal/).

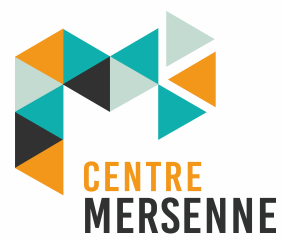

Algebraic Combinatorics is member of the Centre Mersenne for Open Scientific Publishing www.centre-mersenne.org 


\title{
Higher discrete homotopy groups of graphs
}

\author{
Bob Lutz
}

\begin{abstract}
This paper studies a discrete homotopy theory for graphs introduced by Barcelo et al. We prove two main results. First we show that if $G$ is a graph containing no 3- or 4-cycles, then the $n$th discrete homotopy group $A_{n}(G)$ is trivial for all $n \geqslant 2$. Second we exhibit for each $n \geqslant 1$ a natural homomorphism $\psi: A_{n}(G) \rightarrow \mathcal{H}_{n}(G)$, where $\mathcal{H}_{n}(G)$ is the $n$th discrete cubical singular homology group, and an infinite family of graphs $G$ for which $\mathcal{H}_{n}(G)$ is nontrivial and $\psi$ is surjective. It follows that for each $n \geqslant 1$ there are graphs $G$ for which $A_{n}(G)$ is nontrivial.
\end{abstract}

\section{INTRODUCTION}

In [6] a new homotopy theory for simplicial complexes was introduced, motivated by a search for qualitative invariants in the study of complex systems and their dynamics [7]. Given a simplicial complex $K$, an integer $0 \leqslant q \leqslant \operatorname{dim} K$ and a simplex $\sigma_{0} \in K$ of dimension at least $q$, one defines a family of groups

$$
A_{n}^{q}\left(K, \sigma_{0}\right), \quad n \geqslant 1
$$

called the discrete homotopy groups of $K$. In contrast to classical homotopy theory, the groups (1) are defined combinatorially.

For $n=1$, the group (1) is called the discrete fundamental group, and is well understood. There is a discrete analog of the Seifert-van Kampen theorem, and in fact one can construct a cell complex $X$ such that

$$
A_{1}^{q}\left(K, \sigma_{0}\right) \cong \pi_{1}\left(X, x_{0}\right)
$$

where $\pi_{1}\left(X, x_{0}\right)$ is the classical fundamental group for some $x_{0} \in X$. Results like these have enabled computations of $A_{1}^{q}\left(K, \sigma_{0}\right)$ for interesting simplicial complexes, including Coxeter complexes of finite Coxeter groups [8, 9].

For $n>1$, the situation is less clear. The basic tools for computing classical higher homotopy groups, already a difficult problem in general, have no known discrete analogs. In [1] a higher-dimensional version of (2) was obtained, assuming a "plausible" cubical analog of the simplicial approximation theorem. Despite its plausibility, however, the proposed approximation theorem has resisted all attempts at a proof.

This paper studies the problem of computing higher discrete homotopy groups. To begin, we reduce this problem to considering only graphs instead of general simplicial

Manuscript received 30th March 2020, accepted 12th September 2020.

Keywords. Discrete homotopy, discrete singular cubical homology, $A$-theory, Hurewicz theorem.

ACKNOWLEDGEMENTS. Work of the author was supported by NSF grant DMS-1440140 while in residence at the Mathematical Sciences Research Institute in Berkeley, California during the fall 2019 and spring 2020 semesters. 
complexes. Given a graph $G$ and a vertex $v_{0} \in G$, one can define a family of groups $A_{n}\left(G, v_{0}\right)$ similarly to $(1)$. For any triple $\left(K, q, \sigma_{0}\right)$ as above, there is a pair $\left(G, v_{0}\right)$ such that

$$
A_{n}^{q}\left(K, \sigma_{0}\right) \cong A_{n}\left(G, v_{0}\right) .
$$

Namely, $G$ is the graph whose vertices correspond to the maximal simplices of $K$ of dimension at least $q$, and whose edges correspond to the pairs of such simplices sharing a $q$-face. We can therefore restrict our attention to the groups $A_{n}\left(G, v_{0}\right)$ without loss of generality. In particular, we will consider only connected graphs, so we can ignore the base vertex and simply write $A_{n}(G)=A_{n}\left(G, v_{0}\right)$.

We prove two main results. The first is motivated by a singular homology theory for graphs, introduced in [3] as a companion to the discrete homotopy theory described above. The relevant groups $\mathcal{H}_{n}(G)$, called the discrete singular cubical homology groups of $G$, are defined combinatorially. It can be shown that if $G$ contains no 3 - or 4-cycles, then $\mathcal{H}_{n}(G)$ is trivial for all $n \geqslant 2$. This was the main result of [5]. We prove a similar theorem for discrete homotopy.

THEOREM 1.1. If $G$ contains no 3-or 4-cycles, then $A_{n}(G)$ is trivial for all $n \geqslant 2$.

If $G$ contains no 3- or 4-cycles, then it can be shown that $A_{1}(G) \cong \pi_{1}(G)$, where we regard $G$ as both a graph and a topological space. Thus Theorem 1.1 gives a complete picture of the discrete homotopy of such graphs.

Our second result makes progress toward a discrete Hurewicz theorem. Recall that the classical Hurewicz theorem [12] describes, for any path-connected topological space $X$, a map from $\pi_{n}(X)$ to the $n$th homology group of $X$. If $n=1$, then this map is surjective, and its kernel is the commutator subgroup $\left[\pi_{1}(X), \pi_{1}(X)\right]$. If $n>1$ and $\pi_{k}(X)$ is trivial for all $k<n$, then the map is an isomorphism.

In [3] it was shown that there is a surjective map

$$
A_{1}(G) \rightarrow \mathcal{H}_{1}(G)
$$

with kernel $\left[A_{1}(G), A_{1}(G)\right]$, giving a discrete Hurewicz theorem in dimension 1 . We generalize the map (3) to higher dimensions and show that it is surjective in a large number of cases.

THEOREM 1.2. For any graph $G$ and positive integer $n$, there is a natural homomorphism

$$
\psi: A_{n}(G) \rightarrow \mathcal{H}_{n}(G) .
$$

For each $n \geqslant 1$, there is an infinite family of graphs $G$ for which $\psi$ is surjective and $\mathcal{H}_{n}(G)$ is nontrivial.

An important consequence of Theorem 1.2 is the existence of graphs $G$ with nontrivial groups $A_{n}(G)$ for $n \geqslant 2$. To date, no examples of such graphs have appeared in the literature.

Corollary 1.3. For each $n \geqslant 1$ there are graphs $G$ for which $A_{n}(G)$ is nontrivial.

The paper is organized as follows. In Section 2 we give preliminary definitions and results concerning discrete homotopy theory. In Section 3 we prove Theorem 1.1. In Section 4 we describe the basic constructions needed for Theorem 1.2. In Section 5 we recall the definition of discrete cubical singular homology groups and prove Theorem 1.2. In Section 6 we suggest directions for future work. 


\section{BASIC DEFINITIONS}

2.1. Discrete homotopy. In this paper, the term homotopy refers to the discrete homotopy theory for graphs introduced in [6]. By a graph we will mean one that is simple, connected and undirected. We write $v \in G$ if $v$ is a vertex of a graph $G$. If $G_{1}$ and $G_{2}$ are graphs, then we write $f: G_{1} \rightarrow G_{2}$ when $f$ is a function from the vertex set of $G_{1}$ to the vertex set of $G_{2}$. If two vertices $u, v \in G$ are adjacent or equal, then we write $u \simeq v$.

DeFinition 2.1. A function $f: G_{1} \rightarrow G_{2}$ is a graph map if $f(u) \simeq f(v)$ whenever $u \simeq v$.

Let $\mathbb{Z}$ denote the graph with vertex set $\{\ldots,-1,0,1, \ldots\}$ and an edge $i j$ if and only if $|i-j|=1$. Given an integer $m \geqslant 0$, we write $I_{m}$ for the subgraph of $\mathbb{Z}$ induced by $\{0, \ldots, m\}$. We let $G_{1} \times G_{2}$ denote the Cartesian product of graphs $G_{1}$ and $G_{2}$. The following notion of homotopy defines an equivalence relation on graph maps $G_{1} \rightarrow G_{2}$.

Definition 2.2. Two graph maps $f, g: G_{1} \rightarrow G_{2}$ are homotopic if for some $m$ there is a graph map $h: G_{1} \times I_{m} \rightarrow G_{2}$ such that $h(-, 0)=f$ and $h(-, m)=g$. The map $h$ is a homotopy from $f$ to $g$. We write $h_{i}=h(-, i)$ for all $i$, so $h_{0}=f$ and $h_{m}=g$.

Let $\mathbb{Z}^{n}$ denote the $n$-fold Cartesian product of $\mathbb{Z}$, whose vertices are $n$-tuples of integers. For any $r \geqslant 0$, let $\mathbb{Z}_{\geqslant r}^{n} \subseteq \mathbb{Z}^{n}$ be the subgraph induced by all vertices $x \in \mathbb{Z}^{n}$ with $\left|x_{i}\right| \geqslant r$ for some $i$.

Given a function $f: G_{1} \rightarrow G_{2}$, and subgraphs $H_{1} \subseteq G_{1}$ and $H_{2} \subseteq G_{2}$, we write

$$
f:\left(G_{1}, H_{1}\right) \rightarrow\left(G_{2}, H_{2}\right)
$$

if $v \in H_{1}$ implies that $f(v) \in H_{2}$. We will abuse this notation by writing

$$
f:\left(\mathbb{Z}^{n}, \partial \mathbb{Z}^{n}\right) \rightarrow\left(G, v_{0}\right)
$$

if there exists an $r \geqslant 0$ such that $f$ is a function $\left(\mathbb{Z}^{n}, \mathbb{Z}_{\geqslant r}^{n}\right) \rightarrow\left(G, v_{0}\right)$. The minimum such $r$ is called the radius of $f$. We emphasize that $\partial \mathbb{Z}^{n}$ is not defined by itself, but is shorthand for some $\mathbb{Z}_{\geqslant r}^{n}$. The following notion of homotopy, more restrictive than the last, defines an equivalence relation on graph maps $\left(\mathbb{Z}^{n}, \partial \mathbb{Z}^{n}\right) \rightarrow\left(G, v_{0}\right)$.

Definition 2.3. Two graph maps $f, g:\left(\mathbb{Z}^{n}, \partial \mathbb{Z}^{n}\right) \rightarrow\left(G, v_{0}\right)$, are based homotopic if there is a homotopy $h: \mathbb{Z}^{n} \times I_{m} \rightarrow G$ from $f$ to $g$ such that $h_{i}$ is a graph map $\left(\mathbb{Z}^{n}, \partial \mathbb{Z}^{n}\right) \rightarrow\left(G, v_{0}\right)$ for all $i$. The function $h$ is a based homotopy from $f$ to $g$.

Definition 2.4. Fix $v_{0} \in G$. Let $A_{n}(G)$ denote the set of based homotopy classes $[f]$ of graph maps $f:\left(\mathbb{Z}^{n}, \partial \mathbb{Z}^{n}\right) \rightarrow\left(G, v_{0}\right)$. We endow $A_{n}(G)$ with a group structure as follows. If $f$ and $g$ are graph maps $\left(\mathbb{Z}^{n}, \partial \mathbb{Z}^{n}\right) \rightarrow\left(G, v_{0}\right)$ of radii $r_{f}$ and $r_{g}$, respectively, then the product $[f] \cdot[g]$ in $A_{n}(G)$ is the based homotopy class of the map $p:\left(\mathbb{Z}^{n}, \partial \mathbb{Z}^{n}\right) \rightarrow\left(G, v_{0}\right)$ given by

$$
p\left(x_{1}, \ldots, x_{n}\right)= \begin{cases}f\left(x_{1}, \ldots, x_{n}\right) & \text { if } x_{1} \leqslant r_{f}, \\ g\left(x_{1}-\left(r_{f}+r_{g}\right), x_{2}, \ldots, x_{n}\right) & \text { if } x_{1}>r_{f} .\end{cases}
$$

The identity of this operation is the based homotopy class of the constant map $\mathbb{Z}^{n} \rightarrow$ $v_{0}$. We call $A_{n}(G)$ the $n$th discrete homotopy group of $G$.

REMARK 2.5. In [6], working in the more general setting of simplicial complexes, it is shown that the operation in $A_{n}(G)$ is well defined, that it satisfies the group axioms, and that the definition of $A_{n}(G)$ is independent of the base vertex $v_{0}$ when $G$ is connected. It is also shown that the groups $A_{n}(G)$ are abelian for all $n \geqslant 2$. We will not prove these facts here. 


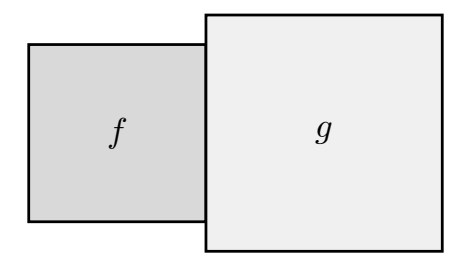

FiguRE 1. Illustrating the group operation in $A_{2}(G)$.

Proposition 2.6 ([6, Proposition 5.12]). Let $G$ be a graph, and let $X_{G}$ be the cell complex obtained by regarding $G$ as a 1-complex and attaching a 2-cell along the boundary of each 3- and 4-cycle. We have $A_{1}(G) \cong \pi_{1}\left(X_{G}\right)$.

EXAmple 2.7. Let $G$ be a cycle graph of length $m$, i.e. $G=\mathbb{Z}_{m}$. If $m=3$ or 4 , then $X_{G}$ is simply connected, so $A_{1}(G)$ is trivial by Proposition 2.6. If $m \geqslant 5$, then $X_{G} \approx S^{1}$, so $A_{1}(G) \cong \mathbb{Z}$. Label the vertices of $G$ in a cycle as $v_{0}, \ldots, v_{m-1}$. An explicit generator of $A_{1}(G)$ is $[f]$, where $f$ is given by $f(i)=v_{i}$ if $0 \leqslant i<m$ and $f(i)=v_{0}$ otherwise.

More generally, suppose that $G$ contains no 3- or 4-cycles. For each cycle $Z$ of $G$ we obtain a generator $\left[f_{Z}\right]$ of $A_{1}(Z)$ as above. A set of generators of $A_{1}(G)$, although not necessarily a minimal one, is the set of $\left[f_{Z}\right]$ for all $Z$.

Example 2.8. Let $K$ be a finite simplicial 2-complex. Suppose, for any vertices $i, j$ and $k$, that if the edges $i j, i k$ and $j k$ all belong to $K$, then so does the triangle $i j k$. Suppose also that the 1-skeleton $K^{1}$ is a chordal graph, i.e. that if a cycle of $G$ is an induced subgraph, then it contains exactly 3 vertices. These conditions ensure that if $G=K^{1}$, then $X_{G}=K$ in the notation of Proposition 2.6, so $A_{1}(G) \cong \pi_{1}(K)$. For example, if $K$ is the triangulation of the real projective plane in Figure 2, then $A_{1}(G) \cong \mathbb{Z} / 2 \mathbb{Z}$. This example generalizes the remark following [6, Proposition 5.12].

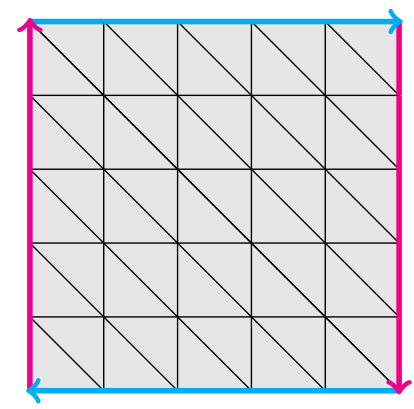

Figure 2. A triangulation of the projective plane.

2.2. Contractibility. We define a notion of a contractible graph as a discrete analog of a contractible topological space. We show that all discrete homotopy groups of a contractible graph are trivial. This fact will be used in proving the main results.

Definition 2.9. A contraction of $G$ is a homotopy from the identity map $G \rightarrow G$ to a constant map. A graph $G$ is contractible if it admits a contraction.

By our definition, infinite graphs are not contractible. We caution that this notion of contractibility does not imply that $\pi_{1}(G)$ is trivial when $G$ is regarded as a topological space. For instance, the next example shows that the square graph $\mathbb{Z}_{4}$ is contractible, but $\pi_{1}\left(\mathbb{Z}_{4}\right) \cong \mathbb{Z}$. 
EXAMPLE 2.10 (Grid graphs). A grid graph is any subgraph $L$ of $\mathbb{Z}^{n}$ induced by a vertex set of the form

$$
\left\{a_{1}, \ldots, b_{1}\right\} \times \cdots \times\left\{a_{n}, \ldots, b_{n}\right\}
$$

for some $a_{i}, b_{i} \in \mathbb{Z}$ with $a_{i} \leqslant b_{i}$ for all $i$. The boundary of $L$ is the subgraph $\partial L$ induced by all vertices $x \in L$ with $x_{i} \in\left\{a_{i}, b_{i}\right\}$ for some $i$. Its complement $L^{\circ}=L \backslash \partial L$ is the interior of $L$. Let $m=\max _{i} b_{i}-a_{i}$, and let $c: L \times I_{m} \rightarrow L$ be given by

$$
c(v, i)=\left(\max \left\{v_{1}-i, 0\right\}, \ldots, \max \left\{v_{n}-i, 0\right\}\right)
$$

for all $(v, i) \in L \times I_{m}$. It is routine to check that $c$ is a homotopy. In particular, $c_{0}$ is the identity map $L \rightarrow L$, and $c_{m}$ is the constant map $L \rightarrow(0, \ldots, 0)$. Hence $L$ is contractible.

EXAmple 2.11 (Finite trees). Suppose that $T$ is a finite tree, and let $v_{0} \in T$. For every $v \in T \backslash v_{0}$, let $\rho(v)$ denote the unique neighbor of $v$ that lies on the simple path from $v_{0}$ to $v$. Set $\rho\left(v_{0}\right)=v_{0}$. This defines a graph map $\rho: G \rightarrow G$. Let $m$ be the diameter of $G$. We define a function $c: G \times I_{m} \rightarrow G$ by setting

$$
c(v, i)= \begin{cases}v & \text { if } i=0 \\ \rho(c(v, i-1)) & \text { if } 1 \leqslant i \leqslant m\end{cases}
$$

for all $v \in T$. It is routine to check that $c$ is a homotopy. In particular, $c_{0}$ is the identity map $T \rightarrow T$ and $c_{m}=v_{0}$, so $G$ is contractible.

Proposition 2.12. If $G$ is contractible, then $A_{n}(G)$ is trivial for all $n$.

Proof. Let $c: G \times I_{m} \rightarrow G$ be a contraction of $G$ to a vertex $v_{0}$, and let $f:\left(\mathbb{Z}^{n}, \partial \mathbb{Z}^{n}\right) \rightarrow$ $\left(G, v_{0}\right)$ be a graph map of radius $r$. If $c\left(v_{0},-\right)$ is constant, then there is an obvious based homotopy $\mathbb{Z}^{n} \times I_{m} \rightarrow G$ from $f$ to the constant map $v_{0}$, given by

$$
(x, i) \mapsto c(f(x), i)
$$

for all $(x, i) \in \mathbb{Z}^{n} \times I_{m}$. However, it need not be the case that $c\left(v_{0},-\right)$ is constant. In general, the map (8) is a homotopy but not a based homotopy, and hence does not preserve the equivalence class $[f] \in A_{n}(G)$.

To get around this, we define a based homotopy $h: \mathbb{Z}^{n} \times I_{2 m} \rightarrow G$ from $f$ to $v_{0}$ in two steps. We first define $h_{i}$ for $i=0, \ldots, m$, and then for $i=m+1, \ldots, 2 m$. Let $\mathbb{Z}_{\leqslant r}^{n} \subseteq \mathbb{Z}^{n}$ denote the grid graph

$$
\mathbb{Z}_{\leqslant r}^{n}=\{-r, \ldots, r\} \times \cdots \times\{-r, \ldots, r\} .
$$

Let $B_{0}=\partial \mathbb{Z}_{\leqslant r}^{n}$. For $k \geqslant 1$, let $B_{k}$ denote the boundary of the grid graph whose interior is $\mathbb{Z}_{\leqslant r}^{n} \cup B_{0} \cup \cdots \cup B_{k-1}$. Note that $\left\{\mathbb{Z}_{\leqslant r}^{n}, B_{1}, B_{2}, \ldots\right\}$ partitions $\mathbb{Z}^{n}$. Let $h_{0}=f$. For $i=1, \ldots, m$ let $h_{i}(x)=c(f(x), i)$ for all $x \in \mathbb{Z}_{\leqslant r}^{n}$, and let $h_{i}$ be constant on $B_{k}$ for each $k \geqslant 1$, taking the same value that $h_{i-1}$ takes on $B_{k-1}$. The result is that $h_{m}$ is constant on $\mathbb{Z}_{\leqslant r}^{n}$ and each $B_{k}$.

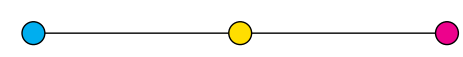

\begin{tabular}{c|c|c|c|c|c}
$v_{v}^{i}$ & 0 & 1 & 2 & 3 & 4 \\
\hline$\circ$ & $\circ$ & $\circ$ & $\circ$ & $\circ$ & $\circ$ \\
\hline$\circ$ & $\circ$ & $\circ$ & $\circ$ & $\circ$ & $\circ$ \\
\hline$\circ$ & $\circ$ & $\circ$ & $\circ$ & $\circ$ & $\circ$
\end{tabular}

Figure 3. A graph $G$ and a table of values of a contraction $c$ : $G \times I_{4} \rightarrow G$.

For example, let $G$ be the graph on the left side of Figure 3, whose vertices are labeled by colors. Let $c: G \times I_{4} \rightarrow v_{0}$ be the contraction of $G$ with values $c(v, i)$ given 
in the table on the right side of Figure 3, so that $m=4$. We can represent a graph map $\mathbb{Z}^{2} \rightarrow G$ as an infinite 2-dimensional array of colored dots, where the color of the dot at $x \in \mathbb{Z}^{2}$ corresponds to the value of the map at $x$. Several such maps appear in Figure 4, where we have restricted our attention to the domain $\mathbb{Z}_{\leqslant 5}^{2}$. We assume that each of these maps takes the color blue everywhere outside the pictured region. Let $f$ be the map on the top left of Figure 4 , and note that $r=2$ is the radius of $f$. Thus $\mathbb{Z}_{\leqslant r}^{n}$ is outlined by dashes. The figure illustrates the maps $h_{i}$ from above for $i=0, \ldots, 4$.

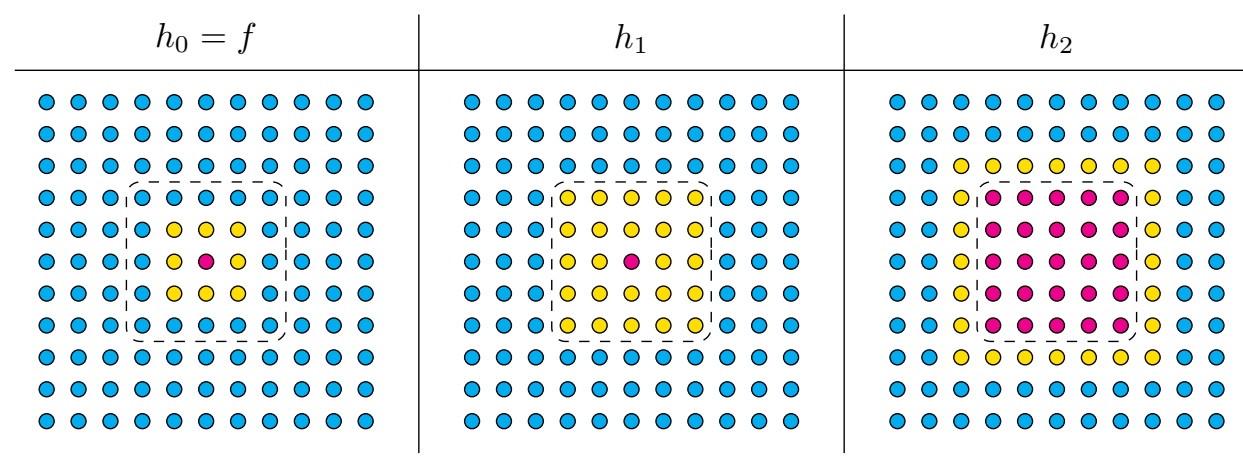

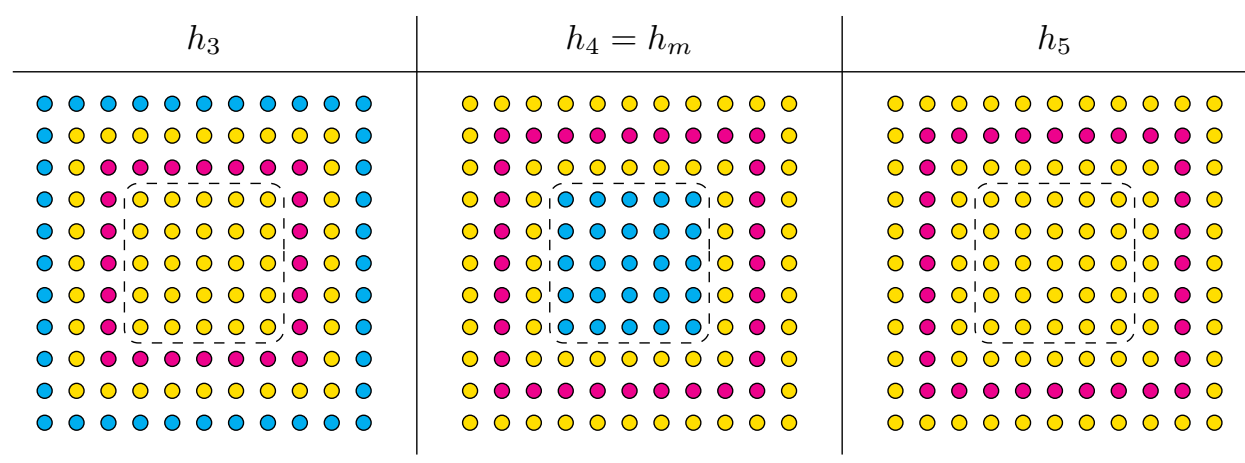

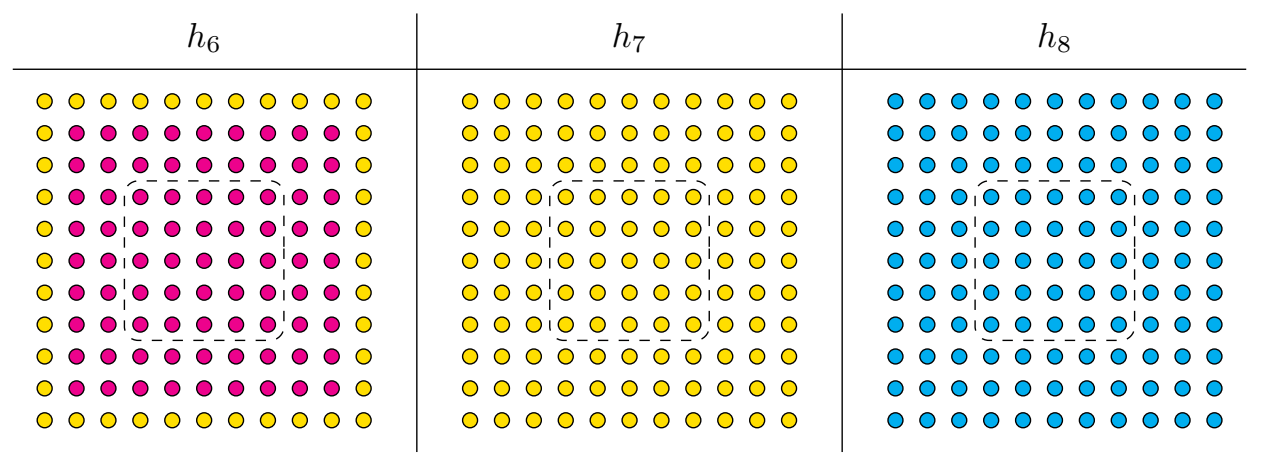

Figure 4 . The stages of a homotopy $h: \mathbb{Z}^{2} \times I_{8} \rightarrow G$.

Returning to the general case, we define $h_{i}$ for the remaining values of $i$. Suppose that $i \in\{m+1, \ldots, 2 m\}$. Let $h_{i}$ be constant on $\mathbb{Z}_{\leqslant r}^{n} \cup B_{m+1} \cup \cdots \cup B_{i-m}$, taking the same value that $h_{m}$ takes on $B_{i-m}$. For $k>i-m$, let $h_{i}$ equal $h_{m}$ on $B_{k}$. Continuing the example from Figure 3, these maps $h_{i}$ are illustrated in Figure 4 for $i=5, \ldots, 8$. In the general case, it is routine to verify that $h: \mathbb{Z}^{n} \times I_{2 m} \rightarrow G$ is a based homotopy; in particular, we have $h_{0}=f$ and $h_{2 m}=v_{0}$. Hence $[f]=0$ in $A_{n}(G)$, as desired. 
If there exists a contraction $c$ of $G$ to $v_{0}$ such that $c\left(v_{0},-\right)$ is constant, then we say that $G$ deformation retracts onto $v_{0}$. In classical topology, there are examples of contractible spaces that do not deformation retract onto a point (see e.g. [11, Chapter 0, Exercise 6] and [2, Section 2]). However, these examples rely on behaviors that have no analogs in the discrete theory. We are led to ask the following.

Question 2.13. Suppose that $G$ is contractible. Does $G$ deformation retract onto a vertex?

\section{TRIANGLE- AND SQUARE-FREE GRAPHS}

We prove Theorem 1.1. If $G$ contains no 3 - or 4 -cycles, then $A_{1}(G) \cong \pi_{1}(G)$ by Proposition 2.6, so we obtain a complete description of the discrete homotopy groups in this case.

Definition 3.1. A path in $G$ is a sequence $\left(p_{1}, \ldots, p_{k}\right)$ of vertices of $G$ such that $p_{i} \simeq p_{i+1}$ for all $i=1, \ldots, k-1$.

Proof of Theorem 1.1. Suppose that $G$ contains no 3 - or 4 -cycles, and set $n \geqslant 2$. Let $f:\left(\mathbb{Z}^{n}, \partial \mathbb{Z}^{n}\right) \rightarrow\left(G, v_{0}\right)$ be a graph map of radius $r$. We show that $[f]=0$ in $A_{n}(G)$. For this proof only, consider $G$ as directed with edge set $E$, so that if $(u, v) \in E$, then $(v, u) \notin E$. Let $F_{E}$ be the free group on $E$, whose elements are the identity 1 and reduced words in the letters $e$ and $e^{-1}$ for all $e \in E$.

We define a function $\tau$ from the set of paths in $\mathbb{Z}^{n}$ of length at least 2 to $F_{E}$ as follows. Given adjacent vertices $x$ ans $y$ of $\mathbb{Z}^{n}$, define an element $\tau(x, y)$ of $F_{E}$ by

$$
\tau(x, y)= \begin{cases}(f(x), f(y)) & \text { if }(f(x), f(y)) \in E \\ (f(y), f(x))^{-1} & \text { if }(f(y), f(x)) \in E \\ 1 & \text { if } f(x)=f(y) .\end{cases}
$$

Given a path $P=\left(p_{0}, \ldots, p_{\ell}\right)$ in $\mathbb{Z}^{n}$ with $\ell>1$, let

$$
\tau(P)=\tau\left(p_{0}, p_{1}\right) \tau\left(p_{1}, p_{2}\right) \cdots \tau\left(p_{\ell-1}, p_{\ell}\right) .
$$

Let $g: \mathbb{Z}^{n} \rightarrow F_{E}$ be given by

$$
g(x)=\tau(P)
$$

where $P$ is any path in $\mathbb{Z}^{n}$ beginning in $\mathbb{Z}_{\geqslant r}^{n}$ and ending with $x$. For example, consider the graph $G$ and the graph map $f:\left(\mathbb{Z}^{2}, \partial \mathbb{Z}^{2}\right) \rightarrow(G, a)$ illustrated in Figure 5, where $r=4$ and $\mathbb{Z}_{\geqslant r}^{n}$ consists of all vertices outside the dashed line. Let $x=(0,-2)$, so that $f(x)=c$. The three highlighted paths from $\mathbb{Z}_{\geqslant r}^{n}$ to $x$ give three ways to compute $g(x)$, all resulting in the same value. Using the green path, clearly $g(x)=(a, b)(b, c)$. For the blue path, we have

$$
g(x)=(a, b)(b, c)(c, d)(c, d)^{-1}(b, c)^{-1}(b, c)=(a, b)(b, c) .
$$

A similar computation with the red path gives the same result. Returning to the general case, it is not clear a priori that $g$ is well defined. We will briefly assume that $g$ is well defined in order to finish the proof. We will then prove our assumption.

Assume that $g$ is well defined, and consider the Cayley graph $\Gamma\left(F_{E}, E\right)$ of $F_{E}$ with generating set $E$. Let $G_{E}$ be the subgraph of $\Gamma\left(F_{E}, E\right)$ induced by

$$
\left\{\tau(P): P \text { is a path in } \mathbb{Z}^{n} \text { beginning in } \mathbb{Z}_{\geqslant r}^{n}\right\} .
$$

Note that $G_{E}$ is a finite tree, since it is a finite connected subgraph of the tree $F_{E}$. The distance between any two vertices $u$ and $v$ in $G_{E}$ is given by $\left|u^{-1} v\right|$, where if $w \in F_{E}$, then $|w|$ denotes the length of the reduced word representing $w$. If $x \simeq y$ in $\mathbb{Z}^{n}$, then $\left|g(x)^{-1} g(y)\right|=|\tau(x, y)| \leqslant 1$, so $g(x) \simeq g(y)$ in $G_{E}$. Hence $g:\left(\mathbb{Z}^{n}, \partial \mathbb{Z}^{n}\right) \rightarrow\left(G_{E}, 1\right)$ is a 

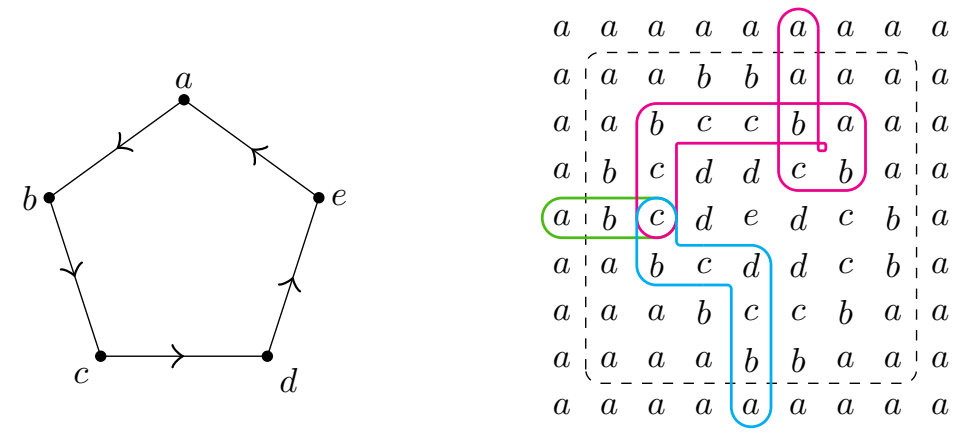

Figure 5. A graph $G=\mathbb{Z}_{5}$ and a graph map $f:\left(\mathbb{Z}^{2}, \partial \mathbb{Z}^{2}\right) \rightarrow(G, a)$.

graph map. Since finite trees are contractible by Example 2.11, Proposition 2.12 gives a based homotopy $h$ from $g$ to the constant map 1 . Every vertex of $G_{E}$ corresponds to a path in $G$ from $v_{0}$ to some vertex; denote this vertex by $\pi(v)$. Clearly $\pi:\left(G_{E}, 1\right) \rightarrow$ $\left(G, v_{0}\right)$ is a graph map and $\pi \circ g=f$, so the composition $\pi \circ h$ is a based homotopy from $f$ to the constant map $v_{0}$. Hence $[f]$ is the identity element in $A_{n}(G)$, proving that $A_{n}(G)$ is trivial.

We now prove that $g$ is well defined. Let $P=\left(p_{0}, \ldots, p_{\ell}\right)$ be a path in $\mathbb{Z}^{n}$. Suppose for some $0<j<\ell$ that the vertices $p_{j-1}, p_{j}$ and $p_{j+1}$ are three corners of a square in $\mathbb{Z}^{n}$. Let $q_{j}$ denote the fourth corner of this square. Consider the operation of replacing the term $p_{j}$ in $P$ with $q_{j}$. We call this operation a corner swap. Next suppose that $p_{k-1}=p_{k+1}$ for some $0<k<\ell$. Consider the operation of removing the terms $p_{k-1}$ and $p_{k}$ from $P$. We call this operation a backtrack deletion.

We show that $\tau(P)$ is invariant under corner swaps and backtrack deletions. Let $p_{j}$ and $q_{j}$ be as above. The vertices $p_{j-1}, p_{j}, p_{j+1}$ and $q_{j}$ form a square in $\mathbb{Z}^{n}$. Since $f$ is a graph map and $G$ contains no squares, the image of this square under $f$ is a path in $G$ consisting of at most 3 vertices. The nontrivial possibilities are illustrated on the right side of Figure 6 up to relabeling. If the top case holds, then by using the definition of $\tau$, we see that

$$
\tau\left(p_{j-1}, p_{j}, p_{j+1}\right)=\tau\left(p_{j}, p_{j+1}\right)=\tau\left(p_{j}, q_{j}, p_{j+1}\right)=\tau\left(p_{j-1}, q_{j}, p_{j+1}\right) .
$$

If the middle case holds, then

$$
\tau\left(p_{j-1}, p_{j}, p_{j+1}\right)=1=\tau\left(p_{j-1}, q_{j}, p_{j+1}\right) .
$$

If the bottom case holds, then $\tau\left(p_{j-1}, p_{j}, p_{j+1}\right)=\tau\left(p_{j-1}, q_{j}, p_{j+1}\right)$ immediately. Hence in any case, we have

$$
\begin{aligned}
\tau(P) & =\tau\left(p_{0}, \ldots, p_{j-1}\right) \tau\left(p_{j-1}, p_{j}, p_{j+1}\right) \tau\left(p_{j+1}, \ldots, p_{\ell}\right) \\
& =\tau\left(p_{0}, \ldots, p_{j-1}\right) \tau\left(p_{j-1}, q_{j}, p_{j+1}\right) \tau\left(p_{j+1}, \ldots, p_{\ell}\right) \\
& =\tau\left(p_{0}, \ldots, p_{j-1}, q_{j}, p_{j+1}, \ldots, p_{\ell}\right),
\end{aligned}
$$

proving that $\tau(P)$ is invariant under corner swaps. To prove that $\tau(P)$ is invariant under backtrack deletions, let $p_{k}$ be as in the previous paragraph, so that $p_{k-1}=p_{k+1}$. We have $\tau\left(p_{k-1}, p_{k}, p_{k+1}\right)=1$, so

as desired.

$$
\begin{aligned}
\tau(P) & =\tau\left(p_{0}, \ldots, p_{k-1}\right) \tau\left(p_{k-1}, p_{k}, p_{k+1}\right) \tau\left(p_{k+1}, \ldots, p_{\ell}\right) \\
& =\tau\left(p_{0}, \ldots, p_{k-1}\right) \tau\left(p_{k+1}, \ldots, p_{\ell}\right) \\
& =\tau\left(p_{0}, \ldots, p_{k-2}, p_{k+1}\right) \tau\left(p_{k+1}, \ldots, p_{\ell}\right) \\
& =\tau\left(p_{0}, \ldots, p_{k-2}, p_{k+1}, \ldots, p_{\ell}\right),
\end{aligned}
$$




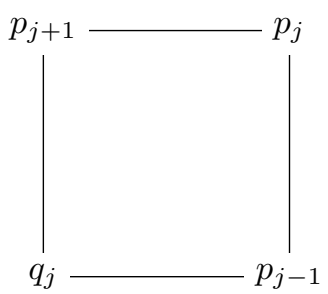

$$
\begin{gathered}
f\left(q_{j}\right)=f\left(p_{j+1}\right) \longrightarrow f\left(p_{j-1}\right)=f\left(p_{j}\right) \\
f\left(p_{j+1}\right) \longleftarrow f\left(p_{j}\right)=f\left(q_{j}\right) \longrightarrow f\left(p_{j-1}\right) \\
f\left(q_{j}\right) \longleftarrow f\left(p_{j-1}\right)=f\left(p_{j+1}\right) \longrightarrow
\end{gathered}
$$

FiguRE 6. A square in $\mathbb{Z}^{n}$ and three possibilities for its image under $f$.

If $P=\left(p_{0}, \ldots, p_{\ell}\right)$ is a closed path in $\mathbb{Z}^{n}$, then it is not hard to see that $P$ can be transformed into the path $\left(p_{0}, p_{1}, p_{0}\right)$ by a sequence of corner swaps and backtrack deletions. Since $\tau(P)$ is invariant under these operations, we must have

$$
\tau(P)=\tau\left(p_{0}, p_{1}, p_{0}\right)=1
$$

whenever $P$ is closed. Let $x \in \mathbb{Z}^{n}$, and let $p, q \in \mathbb{Z}_{\geqslant r}^{n}$. Let $P$ be a path in $\mathbb{Z}^{n}$ from $p$ to $x$ and $Q$ a path from $q$ to $x$. Let $R$ be a path from $q$ to $p$ with all vertices in $\mathbb{Z}_{\geqslant r}^{n}$. Since $f$ is constant on $\mathbb{Z}_{\geqslant r}^{n}$, we have $\tau(R)=1$. Write $P Q^{-1} R$ for the path obtained by concatenating $P$, the reverse of $Q$ and $R$. Since $P Q^{-1} R$ is closed, we have $\tau\left(P Q^{-1} R\right)=1$. Thus

$$
1=\tau\left(P Q^{-1} R\right)=\tau(P) \tau(Q)^{-1} \tau(R)=\tau(P) \tau(Q)^{-1},
$$

giving $\tau(P)=\tau(Q)$. It follows that if a path begins in $\mathbb{Z}_{\geqslant r}^{n}$, its value under $\tau$ depends only on its endpoint. In other words, the function $g$ from (9) is well defined.

\section{Cones And SUSpensions}

We define discrete analogs of the cone and suspension functors from classical topology. This material plays a crucial role in the proof of Theorem 1.2.

Definition 4.1. For $s \geqslant 1$, let $C_{s} G$ denote the graph obtained from $G \times I_{s}$ by contracting the subgraph $G \times\{0\}$. We call $C_{s} G$ a cone on $G$.

Proposition 4.2. The cone $C_{s} G$ on $G$ is contractible for any $s \geqslant 1$.

Proof. Write $C G=C_{s} G$. For each $v \in G$ and $i=1, \ldots, s$, there is a corresponding vertex $v_{i}$ of $C G$ contained in $G \times\{i\}$. We let $v_{0}$ denote the lone vertex in the image of $G \times\{0\}$ in $C G$. Thus the vertices of $C G$ are indexed by $v_{i}$ for $v \in G$ and $i=0, \ldots, n$, with the understanding that $v_{0}=w_{0}$ for all vertices $v$ and $w$ of $G$. This labeling scheme is illustrated in Figure 7. Let $h: C G \times I_{s}$ be given by

$$
c\left(v_{i}, j\right)= \begin{cases}v_{s-j} & \text { if } i>s-j, \\ v_{i} & \text { if } i \leqslant s-j .\end{cases}
$$

It is routine to check that $c$ is a graph map. In addition, $c(-, 0)$ is the identity map on $C G$ and $c(-, s)=v_{0}$. Hence $c$ is a contraction of $C G$.

Definition 4.3. For $t \geqslant 2$, let $S_{t} G$ be the graph obtained from $G \times I_{t}$ by contracting $G \times\{0\}$ and $G \times\{t\}$ to single vertices. We call $S_{t} G$ a suspension of $G$.

Proposition 4.4. If $t=2$ or $G$ is contractible, then $S_{t} G$ is contractible. 

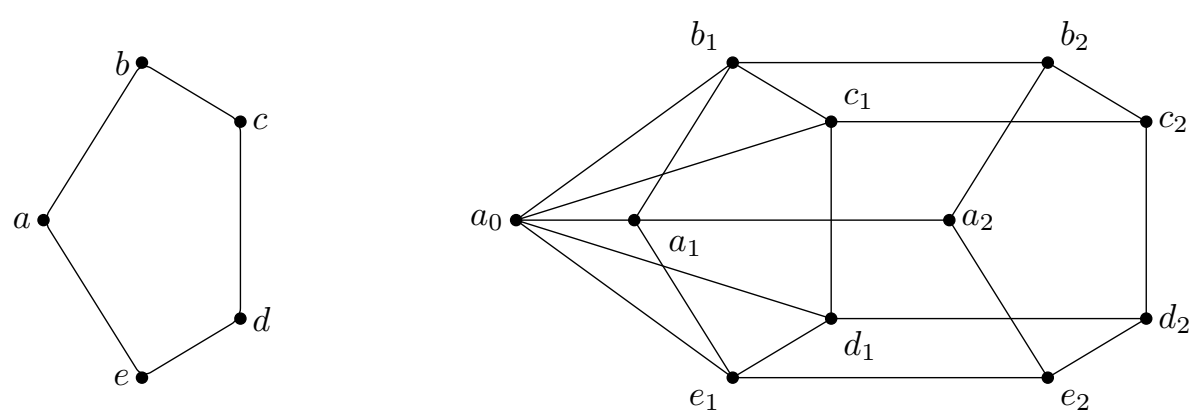

Figure 7 . The pentagon $G=\mathbb{Z}_{5}$, left; and the cone $C_{2} G$, right, with labeling scheme as in the proof of Proposition 4.2.

Proof. We will need a labeling scheme for the vertices of $S_{t} G$. For each $v \in G$ and $i=0, \ldots, t$, we let $v_{i}$ denote the corresponding vertex in the image of $G \times\{i\}$ in $S G$. Thus, for example, $u_{0}=v_{0}$ and $u_{t}=v_{t}$ for all $u, v \in G$. This labeling scheme is illustrated in Figure 8, where the pentagon $G=\mathbb{Z}_{5}$ is labeled as in Figure 7.

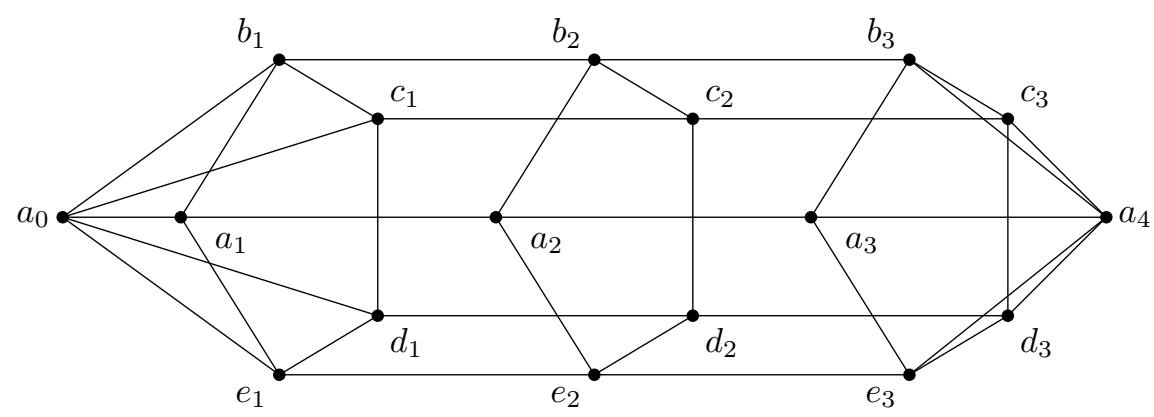

Figure 8. The labeling scheme of $S_{4} \mathbb{Z}_{5}$.

Suppose that $t=2$, and fix $w \in G$. There is contraction $c: S_{t} G \times I_{2} \rightarrow S_{t} G$ of $S_{t} G$ to $v_{0}$ given by

$$
c\left(v_{i}, 1\right)= \begin{cases}v_{0} & \text { if } i<2, \\ w_{1} & \text { if } i=2 .\end{cases}
$$

It is routine to verify that $c$ is a graph map.

Suppose now that $t$ is arbitrary and $G$ is contractible. Let $C: G \times I_{m} \rightarrow G$ be a contraction of $G$ to $w \in G$. Define a function $\kappa: S_{t} G \times I_{m+t} \rightarrow S_{t} G$ as follows:

$$
\kappa\left(v_{i}, j\right)= \begin{cases}C(v, j)_{i} & \text { if } 0 \leqslant j \leqslant m \\ w_{i+m-j} & \text { if } m<j \leqslant i+m, \\ v_{0} & \text { if } i+m<j \leqslant m+t .\end{cases}
$$

It is routine to verify that $C$ is a contraction of $S_{t} G$ to $v_{0}$.

\section{Toward A Discrete Hurewicz Theorem}

We prove Theorem 5.10 below, which implies Theorem 1.2 from the introduction. We first recall the definition of discrete singular cubical homology groups of graphs and define the map $\psi: A_{n}(G) \rightarrow \mathcal{H}_{n}(G)$ from (4). 
5.1. Discrete Singular CubicAl homology of GRAPHs. Let $I_{m}^{n}$ denote the $n$ fold Cartesian product of $I_{m}$, regarded as a subgraph of $\mathbb{Z}^{n}$. For each $n \geqslant 0$, let $Q_{n}$ be the graph given by

$$
Q_{n}= \begin{cases}I_{0} & \text { if } n=0 \\ I_{1}^{n} & \text { if } n \geqslant 1 .\end{cases}
$$

An $n$-cube of $G$ is a graph map $\sigma: Q_{n} \rightarrow G$. For $1 \leqslant i \leqslant n$, let $D_{i}^{-} \sigma$ and $D_{i}^{+} \sigma$ be the $(n-1)$-cubes of $G$ given by

$$
\begin{aligned}
& D_{i}^{-} \sigma\left(a_{1}, \ldots, a_{n-1}\right)=\sigma\left(a_{1}, \ldots, a_{i-1}, 0, a_{i}, \ldots, a_{n-1}\right) \\
& D_{i}^{+} \sigma\left(a_{1}, \ldots, a_{n-1}\right)=\sigma\left(a_{1}, \ldots, a_{i-1}, 1, a_{i}, \ldots, a_{n-1}\right) .
\end{aligned}
$$

We say that $\sigma$ is degenerate if $D_{i}^{-} \sigma=D_{i}^{+} \sigma$ for some $i$. Let $L_{n}(G)$ denote the free $\mathbb{Z}$ module generated by all $n$-cubes of $G$, and let $D_{n}(G)$ denote the submodule generated by the degenerate $n$-cubes of $G$. Let

$$
C_{n}(G)=L_{n}(G) / D_{n}(G) \text {. }
$$

The elements of $C_{n}(G)$ are called $n$-chains of $G$.

Given an $n$-cube $\sigma$ of $G$ with $n \geqslant 1$, let

$$
\partial_{n}(\sigma)=\sum_{i=1}^{n}(-1)^{i}\left(D_{i}^{-} \sigma-D_{i}^{+} \sigma\right) .
$$

Extend linearly to obtain a map $\partial_{n}: C_{n}(G) \rightarrow C_{n-1}(G)$. It is routine to check that $\left(C_{\bullet}(G), \partial_{\bullet}\right)$ is a chain complex. Let

$$
\mathcal{H}_{n}(G)=\operatorname{ker}\left(\partial_{n}\right) / \operatorname{im}\left(\partial_{n+1}\right) .
$$

The group $\mathcal{H}_{n}(G)$ is called the $n$th discrete singular cubical homology group of $G$. If $z \in \operatorname{ker}\left(\partial_{n}\right)$, then we write $\bar{z}$ for the equivalence class of $z$ in $\mathcal{H}_{n}(G)$.

There is a discrete Hurewicz theorem in dimension 1:

TheOREM 5.1 ([3, Theorem 4.1]). For any graph $G$, there is a surjective map

$$
\psi: A_{1}(G) \rightarrow \mathcal{H}_{1}(G)
$$

whose kernel is $\left[A_{1}(G), A_{1}(G)\right]$.

The map $\psi$ can be described explicitly. For this, and for the remainder of this section, we will represent based homotopy classes $[f] \in A_{n}(G)$ as graph maps

$$
\left(I_{m}^{n}, \partial I_{m}^{n}\right) \rightarrow\left(G, v_{0}\right) .
$$

This is justified by the following argument. Let $f:\left(\mathbb{Z}^{n}, \partial \mathbb{Z}^{n}\right) \rightarrow\left(G, v_{0}\right)$ be any graph map of radius $r$. Let $h: \mathbb{Z}^{n} \times I_{r} \rightarrow G$ be given by $h(x, i)=f\left(x_{1}-i, \ldots, x_{n}-i\right)$ for all $(x, i) \in \mathbb{Z}^{n} \times I_{r}$. It is routine to check that $h$ is a based homotopy from $h_{0}=f$ to a graph map $h_{r}$ that is constant outside the interior of $I_{m}^{n}$. By restricting our attention to the domain $I_{m}^{n}$, we obtain a map $\left(I_{m}^{n}, \partial I_{m}^{n}\right) \rightarrow\left(G, v_{0}\right)$ that is based homotopic to $f$.

Following the above, suppose that $f:\left(I_{m}, \partial I_{m}\right) \rightarrow\left(G, v_{0}\right)$ is a graph map, and for $i=0, \ldots, m-1$ let $f^{i}: Q_{1} \rightarrow G$ be given by $f^{i}(0)=f(i)$ and $f^{i}(1)=f(i+1)$. We have

$$
\psi([f])=\sum_{i=0}^{m-1} f^{i} .
$$

For higher dimensions, we conjecture a direct analog of the classical theorem.

Conjecture 5.2. If $n \geqslant 2$ and $A_{k}(G)$ is trivial for all $k<n$, then $A_{n}(G) \cong \mathcal{H}_{n}(G)$. 
EXAMPLE 5.3. Let $G_{1}$ be a graph containing no 3- or 4-cycles. It follows from Proposition 2.6 and [11, Proposition 1A.2] that $A_{1}\left(G_{1}\right)$ is a free group of rank $k$, where $k$ is the number of edges of $G_{1}$ not contained in a given spanning tree. Theorem 5.1 implies that

$$
\mathcal{H}_{1}\left(G_{1}\right) \cong \mathbb{Z}^{k}
$$

where $\mathbb{Z}^{k}$ denotes a free abelian group of rank $k$. An explicit set of generators, although not always minimal one, is the set of $\psi\left(\left[f_{Z}\right]\right)$ for cycles $Z$ of $G_{1}$, where the maps $f_{Z}$ are defined as in Example 2.7. For each $n \geqslant 1$, let $G_{n+1}=S_{n+3} G_{n}$. It will follow from Proposition 5.12(ii) below that $\mathcal{H}_{n}\left(G_{n}\right) \cong \mathbb{Z}^{k}$ for all $n \geqslant 1$. This result originally appeared in [5].

5.2. A proposed discrete Hurewicz map. We define a map

$$
\psi: A_{n}(G) \rightarrow \mathcal{H}_{n}(G)
$$

that generalizes (10). Let $I_{m_{1}, \ldots, m_{n}} \subseteq \mathbb{Z}^{n}$ be the grid graph given by

$$
I_{m_{1}, \ldots, m_{n}}=I_{m_{1}} \times \cdots \times I_{m_{n}} .
$$

For the following definitions, let $L=I_{m_{1}, \ldots, m_{n}}$ and $L^{\prime}=I_{m_{1}-1, \ldots, m_{n}-1}$ for some $m_{i} \geqslant 1$, and let $f: L \rightarrow G$ be a graph map.

Definition 5.4. For each $x \in L^{\prime}$, let $f^{x}: Q_{n} \rightarrow G$ be the graph map given by

$$
f^{x}(y)=f(x+y) .
$$

The maps $f^{x}$ are called the $n$-cubes of $f$. If $x+Q_{n} \subseteq V$ for some $V \subseteq \mathbb{Z}^{n}$, then we say that $f^{x}$ is contained in $V$.

Definition 5.5. Define an element $\phi(f)$ of $C_{n}(G)$ by

$$
\phi(f)=\sum_{x \in L^{\prime}} f^{x} .
$$

In other words, $\phi(f)$ is the sum of all $n$-cubes of $f$.

LEMMA 5.6. We have the following:

(i) If $[f] \in A_{n}(G)$, then $\phi(f) \in \operatorname{ker}\left(\partial_{n}\right)$,

(ii) If $[f]=[g]$ in $A_{n}(G)$, then $\overline{\phi(f)}=\overline{\phi(g)}$.

Proof. First we prove (i). Let $f:\left(I_{m}^{n}, \partial I_{m}^{n}\right) \rightarrow\left(G, v_{0}\right)$ be a graph map. For $i=1, \ldots, n$ let $\Omega_{i}=\left\{x \in I_{m-1}^{n}: x_{i}=0\right\}$. Let $\epsilon_{i} \in \mathbb{Z}^{n}$ be the $i$ th standard basis vector. We have

$$
\partial_{n} \phi(f)=\sum_{i=1}^{n}(-1)^{i} \sum_{x \in \Omega_{i}} \sum_{r=0}^{m-1} D_{i}^{-} f^{x+r \epsilon_{i}}-D_{i}^{+} f^{x+r \epsilon_{i}} .
$$

For each $i$ and each $x \in \Omega_{i}$, the innermost sum telescopes, and the leftover portion

$$
D_{i}^{-} f^{x}-D_{i}^{+} f^{x+(m-1) \epsilon_{i}}
$$

is 0 , since $f$ is constant on $\partial I_{m}^{n}$. This proves (i).

To prove (ii), let $h$ be a homotopy from $f$ to $g$, say $h: I_{m}^{n+1} \rightarrow G$. By another telescoping argument, it is not hard to see that

$$
\partial_{n+1} \phi(h)=(-1)^{n+1}(\phi(f)-\phi(g))
$$

in $C_{n}(G)$, proving (ii).

Definition 5.7. Let $\psi: A_{n}(G) \rightarrow \mathcal{H}_{n}(G)$ be the function given by $\psi([f])=\overline{\phi(f)}$.

Proposition 5.8. The function $\psi: A_{n}(G) \rightarrow \mathcal{H}_{n}(G)$ is a group homomorphism. 
Proof. If $[f]$ and $[g]$ are any two elements of $A_{n}(G)$, and $p$ is defined as in (7), then $\phi(p)-(\phi(f)+\phi(g))$ is a sum of constant $n$-cubes $Q^{n} \rightarrow v_{0}$, which are degenerate. Hence $\psi([f] \cdot[g])=\psi([p])=\psi([f])+\psi([g])$ in $\mathcal{H}_{n}(G)$, as desired.

REMARK 5.9. If we regard $A_{n}$ (resp. $\mathcal{H}_{n}$ ) as a functor from the category of connected simple graphs with graph maps to the category of groups (resp. abelian groups), then $\psi$ is a natural transformation from $A_{n}$ to $\mathcal{H}_{n}$.

5.3. Surjectivity in a special Case. Given any $n \geqslant 1$, we describe an infinite family of graphs $G$ for which the map $\psi: A_{n}(G) \rightarrow \mathcal{H}_{n}(G)$ is surjective. From this we obtain an infinite class of $G$ for which $A_{n}(G)$ is nontrivial. Throughout the section, we let $G_{1}$ be a graph and

$$
G_{n+1}=S_{n+3} G_{n}
$$

for all $n \geqslant 1$, where $S_{n+3}$ is the suspension functor from Definition 4.3. We think of $G_{n+1}$ as consisting of $n+2$ copies of $G_{n}$ and two extra vertices, called the north pole and south pole. We now state the main result of this section, which implies Theorem 1.2.

THEOREM 5.10. If $G_{1}$ contains no 3- or 4-cycles, then the map

$$
\psi: A_{k}\left(G_{n}\right) \rightarrow \mathcal{H}_{k}\left(G_{n}\right)
$$

is surjective for all $1 \leqslant k \leqslant n$.

Corollary 5.11. If $G_{1}$ contains a cycle but does not contain any 3-or 4-cycles, then $A_{n}\left(G_{n}\right)$ is infinite for all $n \geqslant 1$.

Proof. This follows from Example 5.3, Theorem 5.10 and Proposition 5.12(ii) below.

The proof of Theorem 5.10 will proceed in several steps. We will focus on the case where $G_{1}$ is a cycle graph of length 5 , i.e. $G_{1}=\mathbb{Z}_{5}$, and then extend the proof to the general case. Example 5.3 says that if $G_{1}=\mathbb{Z}_{5}$, then $\mathcal{H}_{n}\left(G_{n}\right) \cong \mathbb{Z}$ for all $n \geqslant 1$. The following proposition, which applies to any choice of $G_{1}$, will allow us to identify an explicit generator of $\mathcal{H}_{n}\left(G_{n}\right)$ when $G_{1}=\mathbb{Z}_{5}$. Part (ii) first appeared as [5, Theorem 5.2].

Proposition 5.12. If $n \geqslant 2$, then

(i) $\mathcal{H}_{k}\left(G_{n}\right)=0$ for $k=1, \ldots, n-1$,

(ii) there is an explicit isomorphism $\Delta: \mathcal{H}_{n}\left(G_{n}\right) \rightarrow \mathcal{H}_{n-1}\left(G_{n-1}\right)$.

Proof. We first prove (ii), adapting our argument from the proof of [5, Theorem 5.2]. Let $n \geqslant 2$. Let $X$ (resp. $Y$ ) be the graph obtained from $G_{n}$ by deleting the north (resp. south) pole. From the proof of [5, Theorem 5.2] we have the following exact sequences for $k=1, \ldots, n$ :

$$
\mathcal{H}_{k}(X) \oplus \mathcal{H}_{k}(Y) \rightarrow \mathcal{H}_{k}\left(G_{n}\right) \stackrel{\partial_{*}}{\longrightarrow} \mathcal{H}_{k-1}(X \cap Y) \rightarrow \mathcal{H}_{k-1}(X) \oplus \mathcal{H}_{k-1}(Y) .
$$

The map $\partial_{*}$ can be described explicitly: if $\bar{z} \in \mathcal{H}_{k}\left(G_{n}\right)$, then $z=x+y$ for some $x \in C_{k}(X)$ and $y \in C_{k}(Y)$, and $\partial_{*}(\bar{z})=\overline{\partial_{k}(x)}$. Note that $G_{n-1} \times\{1\}$ is a deformation retract of $X \cap Y$ in the sense of [4, Section 4]. Hence if we identify $G_{n-1}$ with $G_{n-1} \times\{1\}$, then the inclusion map $\iota: G_{n-1} \rightarrow X \cap Y$ induces an isomorphism $\iota_{*}: \mathcal{H}_{n-1}\left(G_{n-1}\right) \rightarrow \mathcal{H}_{n-1}(X \cap Y)$. Let

$$
\Delta:=\left(\iota_{*}\right)^{-1} \circ \partial_{*} .
$$


Proposition 4.2 implies that $X$ and $Y$ are contractible, so $\mathcal{H}_{k}(X)=\mathcal{H}_{k}(Y)=0$ for all $k \geqslant 1$. The exact sequences (15) now become

$$
0 \rightarrow \mathcal{H}_{k}\left(G_{n}\right) \stackrel{\Delta}{\longrightarrow} \mathcal{H}_{k-1}\left(G_{n-1}\right) \rightarrow \mathcal{H}_{k-1}(X) \oplus \mathcal{H}_{k-1}(Y) .
$$

For $k \geqslant 2$ we have $\mathcal{H}_{k-1}(X) \oplus \mathcal{H}_{k-1}(Y)=0$, so $\Delta$ is an isomorphism, proving (ii).

We now prove (i). If $G=(V, E)$ is any connected graph, then $\mathcal{H}_{0}(G) \cong \mathbb{Z}$ has presentation

$$
\left.\mathcal{H}_{0}(G)=\langle v \in V| u=v \text { for all } u, v \in V\right\rangle .
$$

Any $x \in C_{1}(X)$ can be written as $x=\sum_{i=1}^{\ell} m_{i} f_{i}$ for integers $m_{i}$ and graph maps $f_{i}: Q_{1} \rightarrow X$. We have $\partial_{1}(x)=\sum_{i=1}^{\ell} m_{i}\left(f_{i}(1)-f_{i}(0)\right)$, so $\overline{\partial_{1}(x)}=\sum_{i=1}^{\ell} m_{i}\left(f_{i}(1)-\right.$ $\left.f_{i}(1)\right)=0$ in $\mathcal{H}_{0}\left(G_{n-1}\right)$ by $(16)$. Thus when $k=1$, we have $\operatorname{im} \Delta=0$. But also ker $\Delta=0$ by exactness, so $\mathcal{H}_{1}\left(G_{n}\right)=0$ for all $n \geqslant 2$. Hence if $k<n$, then by applying $(i)$ repeatedly we obtain an isomorphism $\mathcal{H}_{k}\left(G_{n}\right) \rightarrow \mathcal{H}_{1}\left(G_{n-k+1}\right)=0$, proving (i).

We will need a labeling scheme for the vertices of $G_{n}$. The graph $G_{n+1}$ consists of $n+2$ isomorphic copies of $G_{n}$ suspended between the north and south poles. For each $i=0, \ldots, n+3$ we obtain a graph map $\iota_{n}^{i}: G_{n} \rightarrow G_{n+1}$ taking each $v \in G_{n}$ to the corresponding vertex in the $i$ th copy of $G_{n}$. Thus $\iota_{n}^{0}$ and $\iota_{n}^{n+3}$ are the constant graph maps taking $G_{n}$ to the north and south poles of $G_{n+1}$, respectively. For $v \in G_{n}$, we write $v_{i}=\iota_{n}^{i}(v)$ for all $i$, and we say that $v_{i}$ is obtained from $v$ by adding the subscript $i$. In general, if $v$ is a vertex of $G_{k}$ for $k<n$, then we write

$$
v_{i_{k}, \ldots, i_{n}}=\iota_{n}^{i_{n}} \cdots \iota_{k}^{i_{k}} v
$$

For example, $v_{i_{k}, \ldots, i_{n}}$ is the south pole of $G_{n+1}$ if and only if $i_{n}=0$.

If $G$ is a graph and $L=I_{m_{1}, \ldots, m_{n}}$ is a grid graph, then we will think of a map $L \rightarrow G$ as an $n$-dimensional array of size $\left(m_{1}+1\right) \times \cdots \times\left(m_{n}+1\right)$, whose entries are vertices of $G$. For example, when $n=1$, a map $L \rightarrow G$ is a single column with $m_{1}+1$ entries. When $n=2$, we get an $\left(m_{1}+1\right) \times\left(m_{2}+1\right)$ matrix. In both cases, we consider the top-left entry to be the image of the origin $(0, \ldots, 0) \in L$.

Suppose that $G_{1}=\mathbb{Z}_{5}$. Our task now is to construct a generator of $\mathcal{H}_{n}\left(G_{n}\right) \cong \mathbb{Z}$ inductively. Define grid graphs $J_{n}$ by $J_{1}=I_{5}$ and

$$
J_{n+1}=J_{n} \times I_{n+3}
$$

for all $n \geqslant 1$. Label the vertices of $G_{1}$ as in Figure 7. As just described, we will think of maps $J_{n} \rightarrow G$ as $n$-dimensional arrays of appropriate size. Let $\gamma_{1}: J_{1} \rightarrow G_{1}$ be given by

$$
\gamma_{1}=(a b c d e a)^{T}
$$

where $T$ denotes the usual transpose. For $n \geqslant 1$ let $\gamma_{n+1}: J_{n+1} \rightarrow G_{n+1}$ be given by

$$
\gamma_{n+1}(v, i)=\gamma_{n}(v)_{i}
$$

for all $(v, i) \in J_{n} \times I_{n+3}$, where we have added the subscript $i$ to $\gamma_{n}(v)$ as defined in (17). For example, $\gamma_{2}: I_{5} \times I_{4} \rightarrow G$ is given by

$$
\gamma_{2}=\left(\begin{array}{llllll}
a_{0} & a_{0} & a_{0} & a_{0} & a_{0} & a_{0} \\
a_{1} & b_{1} & c_{1} & d_{1} & e_{1} & a_{1} \\
a_{2} & b_{2} & c_{2} & d_{2} & e_{2} & a_{2} \\
a_{3} & b_{3} & c_{3} & d_{3} & e_{3} & a_{3} \\
a_{4} & a_{4} & a_{4} & a_{4} & a_{4} & a_{4}
\end{array}\right)^{T}
$$

It is routine to verify that $\gamma_{n}: J_{n} \rightarrow G_{n}$ is a graph map for all $n$. 
We record some basic properties of $\gamma_{n}$. First, since $g_{1}(0)=g_{1}(5)$, we have

$$
\gamma_{n}\left(0, v_{2}, \ldots, v_{n}\right)=\gamma_{n}\left(5, v_{2}, \ldots, v_{n}\right)
$$

for all $v \in J_{n}$. We also have

$$
\gamma_{n}\left(v_{1}, \ldots, v_{i-1}, 0, v_{i+1}, \ldots, v_{n}\right)=\gamma_{n}\left(0, \ldots, 0, v_{i+1}, \ldots, v_{n}\right)
$$

for all $i=2, \ldots, n$, since adding the subscript 0 to any vertex of $G_{i-1}$ gives the south pole of $G_{i}$. Similarly, adding the subscript $i+2$ to any vertex of $G_{i-1}$ gives the north pole of $G_{i}$, so

$$
\gamma_{n}\left(v_{1}, \ldots, v_{i-1}, i+2, v_{i+1}, \ldots, v_{n}\right)=\gamma_{n}\left(0, \ldots, 0, i+2, v_{i+1}, \ldots, v_{n}\right) .
$$

Finally, note that $\partial_{n} \phi\left(\gamma_{n}\right)=0$ by a telescoping argument, so we can consider the homology class $\overline{\phi\left(\gamma_{n}\right)}$.

LEMMA 5.13. If $G_{1}=\mathbb{Z}_{5}$ and $\gamma_{n}$ is defined as above for $n \geqslant 1$, then

(i) $\mathcal{H}_{n}\left(G_{n}\right)=\left\langle\overline{\phi\left(\gamma_{n}\right)}\right\rangle$

(ii) $\Delta\left(\overline{\phi\left(\gamma_{n+1}\right)}\right)=(-1)^{n} \overline{\phi\left(\gamma_{n}\right)}$, where $\Delta: \mathcal{H}_{n+1}\left(G_{n+1}\right) \rightarrow \mathcal{H}_{n}\left(G_{n}\right)$ is the isomorphism from Proposition 5.12(ii).

Proof. We prove (ii). Example 5.3 implies (i) for $n=1$, so the general case of (i) will follow from (ii). Suppose that $n \geqslant 2$. We retain the definitions of $X, Y, \partial_{*}, \iota$ and $\iota_{*}$ from the proof of Proposition 5.12. Thus $X$ (resp. $Y$ ) is obtained from $G_{n}$ by removing the north (resp. south) pole. We can write $\phi\left(\gamma_{n}\right)=x+y$, where $x \in C_{n}(X)$ and $y \in C_{n}(Y)$. In particular, if $U=\left\{u \in J_{n}: 1 \leqslant u_{n} \leqslant n+1\right\}$, then we can take

$$
y=\sum_{u \in U} \gamma_{n}^{u}
$$

Let $V=\left\{v \in J_{n}: v_{n}=1\right\}$ and $W=\left\{w \in J_{n}: w_{n}=n+1\right\}$. The only terms in the sum $\partial_{n}(y)$ that do not cancel by telescoping are

$$
\partial_{n}(y)=(-1)^{n}\left(\sum_{v \in V} D_{n}^{-} \gamma_{n}^{v}-\sum_{w \in W} D_{n}^{+} \gamma_{n}^{w}\right)
$$

By construction, each $D_{n}^{+} \gamma_{n}^{w}$ is the constant map $Q^{n-1} \rightarrow a_{n+2, \ldots, n+2}$. This is a degenerate $(n-1)$-cube of $G_{n}$, so we can ignore it. We are left with the sum

$$
\partial_{n}(y)=(-1)^{n} \sum_{v \in V} D_{n}^{-} \gamma_{n}^{v}
$$

but this is precisely $\phi\left(\iota \circ \gamma_{n-1}\right)$. Hence

$$
\partial_{*}\left(\overline{\phi\left(\gamma_{n}\right)}\right)=\overline{\partial_{n}(x)}=-\overline{\partial_{n}(y)}=(-1)^{n-1} \overline{\phi\left(\iota \circ \gamma_{n-1}\right)}=(-1)^{n-1}\left(\iota_{*}\left(\overline{\phi\left(\gamma_{n-1}\right)}\right)\right),
$$

from which we deduce that $\Delta\left(\overline{\phi\left(\gamma_{n}\right)}\right)=\left(\iota_{*}\right)^{-1}\left(\partial_{*}\left(\overline{\phi\left(\gamma_{n}\right)}\right)\right)=(-1)^{n-1} \overline{\phi\left(\gamma_{n-1}\right)}$, proving (ii).

LEMMA 5.14. If $G_{1}=\mathbb{Z}_{5}$, then the image of $\psi: A_{n}\left(G_{n}\right) \rightarrow \mathcal{H}_{n}\left(G_{n}\right)$ contains $\overline{\phi\left(\gamma_{n}\right)}$.

We illustrate the proof of Lemma 5.14 for $n=2$ before arguing the general case. Let $L_{2}=I_{13,8}$, and let $M_{2}=(4,0)+I_{5,4} \subseteq L_{2}$. Let $f_{2}:\left(L_{2}, \partial L_{2}\right) \rightarrow\left(G_{2}, a_{0}\right)$ be the graph map in Figure 9. Here $f_{2}$ is constant on every outlined region.

Let $g_{2}:\left(L_{2}, \partial L_{2}\right) \rightarrow\left(G_{2}, a_{0}\right)$ be the graph map in Figure 10 . Here $M_{2}$ is the region outlined in red. Note that $f_{2}(x)=g_{2}(x)$ for all $x \in L_{2} \backslash M_{2}^{\circ}$. Hence if $\Omega_{2}=\left\{x \in \mathbb{Z}^{2}\right.$ : $\left.x+Q_{2} \subseteq M_{2}\right\}$, then

$$
\phi\left(g_{2}\right)-\phi\left(f_{2}\right)=\sum_{x \in \Omega_{2}} g_{2}^{x}-\sum_{x \in \Omega_{2}} f_{2}^{x} .
$$




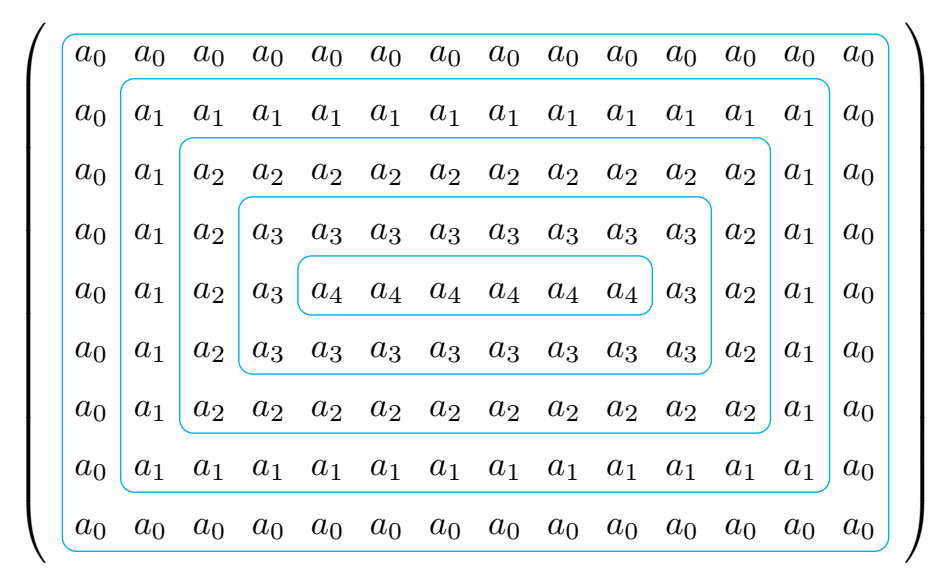

Figure 9. The transpose of $f_{2}$ with its fibers $B_{2, k}$ outlined in blue.

The first sum on the right hand side is easily seen to be $\phi\left(\gamma_{2}\right)$ by inspection. Each term in the second sum is a degenerate 2-cube of $f_{2}$. Hence $\phi\left(g_{2}\right)-\phi\left(f_{2}\right)=\phi\left(\gamma_{2}\right)$, proving that

$$
\psi\left(\left[g_{2}\right]-\left[f_{2}\right]\right)=\overline{\phi\left(\gamma_{2}\right)}
$$

as desired.

$$
\left(\begin{array}{ccccc|cccccc|cccc}
a_{0} & a_{0} & a_{0} & a_{0} & a_{0} & a_{0} & a_{0} & a_{0} & a_{0} & a_{0} & a_{0} & a_{0} & a_{0} & a_{0} \\
a_{0} & a_{1} & a_{1} & a_{1} & a_{1} & b_{1} & c_{1} & d_{1} & e_{1} & a_{1} & a_{1} & a_{1} & a_{1} & a_{0} \\
a_{0} & a_{1} & a_{2} & a_{2} & a_{2} & b_{2} & c_{2} & d_{2} & e_{2} & a_{2} & a_{2} & a_{2} & a_{1} & a_{0} \\
a_{0} & a_{1} & a_{2} & a_{3} & a_{3} & b_{3} & c_{3} & d_{3} & e_{3} & a_{3} & a_{3} & a_{2} & a_{1} & a_{0} \\
a_{0} & a_{1} & a_{2} & a_{3} & a_{4} & a_{4} & a_{4} & a_{4} & a_{4} & a_{4} & a_{3} & a_{2} & a_{1} & a_{0} \\
a_{0} & a_{1} & a_{2} & a_{3} & a_{3} & a_{3} & a_{3} & a_{3} & a_{3} & a_{3} & a_{3} & a_{2} & a_{1} & a_{0} \\
a_{0} & a_{1} & a_{2} & a_{2} & a_{2} & a_{2} & a_{2} & a_{2} & a_{2} & a_{2} & a_{2} & a_{2} & a_{1} & a_{0} \\
a_{0} & a_{1} & a_{1} & a_{1} & a_{1} & a_{1} & a_{1} & a_{1} & a_{1} & a_{1} & a_{1} & a_{1} & a_{1} & a_{0} \\
a_{0} & a_{0} & a_{0} & a_{0} & a_{0} & a_{0} & a_{0} & a_{0} & a_{0} & a_{0} & a_{0} & a_{0} & a_{0} & a_{0} \\
& & & & & & & & & & & & &
\end{array}\right)
$$

Figure 10. The transpose of $g_{2}$ with $M_{2}$ outlined in red and each $B_{2, k} \backslash M_{2}$ outlined in blue.

Proof of Lemma 5.14. The case $n=1$ is trivial; simply note that

$$
\psi\left(\left[\gamma_{1}\right]\right)=\overline{\phi\left(\gamma_{1}\right)}
$$

Suppose now that $n \geqslant 2$. We will construct an $n$-dimensional grid graph $L_{n}$ and graph maps

$$
f_{n}, g_{n}:\left(L_{n}, \partial L_{n}\right) \rightarrow\left(G_{n}, a_{0, \ldots, 0}\right)
$$

such that

$$
\psi\left(\left[g_{n}\right]-\left[f_{n}\right]\right)=\overline{\phi\left(\gamma_{n}\right)}
$$

proving the result. 
For $n \geqslant 2$ let $L_{n}=I_{m_{1}, \ldots, m_{n}}$, where

$$
m_{i}= \begin{cases}(n-i+1)(n+i+4)-1 & \text { if } i=1 \\ (n-i+1)(n+i+4) & \text { if } i>1\end{cases}
$$

For example, the first several $L_{n}$ are

Also let

$$
\begin{aligned}
& L_{2}=I_{13} \times I_{8} \\
& L_{3}=I_{23} \times I_{18} \times I_{10} \\
& L_{4}=I_{35} \times I_{30} \times I_{22} \times I_{12} .
\end{aligned}
$$

$$
M_{n}=c+J_{n} \subseteq L_{n},
$$

where $c=(n+2, \ldots, n+2,0) \in \mathbb{Z}^{n}$ and $J_{n}$ is defined as in (18). Let $L_{n, 0}=\partial L_{n}$, and for $k=1, \ldots, n+2$ let $L_{n, k}=L_{n, k-1}^{\circ}$. For each $k=0, \ldots, n+2$, let $B_{n, k}=\partial L_{n, k}$. For example, the graphs $B_{2, k}$ are outlined in Figure 9 , and $M_{2}$ is the large outlined region in Figure 10. Note that the $B_{n, k}$ partition $L_{n}$ in a particular way; there is a "central" grid graph $B_{n, n+2}$ induced by all vertices of $M_{n}$ with $n$th coordinate $n+2$, and $B_{n, k}$ is "wrapped around" $B_{n, k+1}$ for $k=0, \ldots, n+1$.

Suppose that $x \in B_{n, k}$. Let $f_{n}:\left(L_{n}, \partial L_{n}\right) \rightarrow\left(G_{n}, a_{0, \ldots, 0}\right)$ be given by

$$
f_{n}(x)=\gamma_{n}\left(0, x_{2}^{*}, x_{3}^{*}, \ldots, x_{n-1}^{*}, k\right),
$$

where for $1<i<n$ we set

$$
x_{i}^{*}= \begin{cases}x_{i}-(n+2) & \text { if } n+2 \leqslant x_{i} \leqslant n+i+4, \\ 0 & \text { if } x_{i} \leqslant n+2 \\ i+2 & \text { if } x_{i} \geqslant n+i+4 .\end{cases}
$$

For example, $f_{2}$ is illustrated in Figure 9.

We claim that $f_{n}$ is a graph map. Let $x, y \in L_{n}$ with $x \simeq y$. We must show that $f_{n}(x) \simeq f_{n}(x+y)$. If $x=y$, then this is immediate. Suppose that $x \neq y$ with $x \in B_{n, k}$. The vertices $x$ and $y$ differ in exactly one coordinate; call it $j$. We must have either $y \in B_{n, k}$ or $y \in B_{n, k \pm 1}$. If $j \in\{1, n\}$ and $y \in B_{n, k}$, then $f_{n}(x)=f_{n}(y)$ by definition. If $j \in\{1, n\}$ and $y \in B_{n, k \pm 1}$, then

$$
f_{n}(x)=\gamma_{n}\left(0, x_{2}^{*}, \ldots, x_{n-1}^{*}, k\right) \simeq \gamma_{n}\left(0, x_{2}^{*}, \ldots, x_{n-1}^{*}, k \pm 1\right)=f_{n}(y) .
$$

If $1<j<n$ and $y \in B_{n, k}$, then $\left|x_{j}^{*}-y_{j}^{*}\right| \leqslant 1$, so

$$
f_{n}(x)=\gamma_{n}\left(0, x_{2}^{*}, \ldots, x_{n-1}^{*}, k\right) \simeq \gamma_{n}\left(0, x_{2}^{*}, \ldots, x_{j-1}^{*}, y_{j}^{*}, x_{j+1}^{*}, \ldots, x_{n-1}^{*}, k\right)=f_{n}(y) .
$$

If $1<j<n$ and $y \in B_{n, k \pm 1}$, then either $x_{j}, y_{j} \leqslant n+2$ or $x_{j}, y_{j} \geqslant n+j+4$, so $x_{j}^{*}=y_{j}^{*}$ and $(25)$ holds again. Thus in any case we have $f_{n}(x) \simeq f_{n}(y)$, so $f_{n}$ is a graph map.

We claim that the following defines a function $g_{n}:\left(L_{n}, \partial L_{n}\right) \rightarrow\left(G_{n}, a_{0, \ldots, 0}\right)$ :

$$
g_{n}(x)= \begin{cases}\gamma_{n}(x-c) & \text { if } x \in M_{n}, \\ f_{n}(x) & \text { if } x \in L_{n} \backslash M_{n}^{\circ} .\end{cases}
$$

For example, $g_{2}$ is illustrated in Figure 10 with $M_{2}$ boxed in red and $B_{2, k} \backslash M_{2}$ boxed in blue for $k=0, \ldots, 3$. To prove that $g_{n}$ is well defined in general, we must show that

$$
\gamma_{n}(x-c)=f_{n}(x)
$$

for all $x \in \partial M_{n}$. It will follow that $g_{n}$ is a graph map, since $\gamma$ and $f_{n}$ are graph maps.

We have $x \in M_{n}$ if and only if the following hold:

(i) $n+2 \leqslant x_{1} \leqslant n+7$ 
(ii) $n+2 \leqslant x_{i} \leqslant n+i+4$ for $i=2, \ldots, n-1$

(iii) $0 \leqslant x_{n} \leqslant n+2$.

Thus $x \in \partial M_{n}$ if and only if (i)-(iii) hold with at least one of these inequalities being an equality. Note that the graphs $B_{n, k} \cap \partial M_{n}$ partition $\partial M_{n}$. Suppose that $x \in B_{n, k} \cap \partial M_{n}$. We have $x_{n}=k$ and $x_{i}^{*}=x_{i}-(n+2)$ for $1<i<n$, so

$$
\gamma_{n}(x-c)=\gamma_{n}\left(x_{1}-(n+2), x_{2}^{*}, \ldots, x_{n-1}^{*}, k\right) .
$$

If $x_{1}=n+2$, then

$$
\gamma_{n}(x-c)=\gamma_{n}\left(0, x_{2}^{*}, \ldots, x_{n-1}^{*}, k\right)=f_{n}(x)
$$

by definition. If $x_{1}=n+7$, then

$$
\gamma_{n}(x-c)=\gamma_{n}\left(5, x_{2}^{*}, \ldots, x_{n-1}^{*}, k\right)=\gamma_{n}\left(0, x_{2}^{*}, \ldots, x_{n-1}^{*}, k\right)=f_{n}(x),
$$

where we have used (21). If $x_{i}=n+2$ for some $1<i<n$, then $x_{i}^{*}=0$, so

$$
\begin{aligned}
\gamma_{n}(x-c) & =\gamma_{n}\left(x_{1}-(n+2), x_{2}^{*}, \ldots, x_{i-1}^{*}, 0, x_{i+1}^{*}, \ldots, x_{n-1}^{*}, k\right) \\
& =\gamma_{n}\left(0, \ldots, 0, x_{i+1}^{*}, \ldots, x_{n-1}^{*}, k\right) \\
& =\gamma_{n}\left(0, x_{2}^{*}, \ldots, x_{i-1}^{*}, 0, x_{i+1}^{*}, \ldots, x_{n-1}^{*}, x_{n}\right) \\
& =\gamma_{n}\left(0, x_{2}^{*}, \ldots, x_{n-1}^{*}, k\right) \\
& =f_{n}(x)
\end{aligned}
$$

where we have used (22). The argument is similar for $x_{i}=n+i+4$, using (23) instead. If $x_{n}=0$ (resp. $x_{n}=n+2$ ), then (22) (resp. (23)) again implies (26). Therefore (26) holds for all $x \in \partial M_{n}$, proving the claim that $g_{n}$ is well defined. It follows that $g_{n}$ is a graph map.

We now prove (24). Let

$$
\Omega_{n}=\left\{x \in \mathbb{Z}^{n}: x+Q_{n} \subseteq M_{n}\right\} .
$$

Since $g_{n}(x)=f_{n}(x)$ for all $x \in L_{n} \backslash M_{n}^{\circ}$, we have

$$
\phi\left(\left[g_{n}\right]-\left[f_{n}\right]\right)=\sum_{x \in \Omega_{n}} g_{n}^{x}-\sum_{x \in \Omega_{n}} f_{n}^{x} .
$$

The first sum on the right hand side is easily seen to be $\phi\left(\gamma_{n}\right)$. We claim that every term of the second sum is a degenerate $n$-cube of $f_{n}$. If $x \in \Omega_{n}$, then $n+2 \leqslant x_{i}<$ $n+i+4$ for all $i<n$, so for any $q \in Q_{n}$ we have

$$
\begin{aligned}
f_{n}^{x}\left(0, q_{2}, \ldots, q_{n}\right) & =\gamma_{n}\left(0, x_{2}+q_{2}-(n+2), \ldots, x_{n-1}+q_{n-1}-(n+2), x_{n}+q_{n}\right) \\
& =f_{n}^{x}\left(1, q_{2}, \ldots, q_{n}\right) .
\end{aligned}
$$

Hence $D_{1}^{-} f_{n}^{x}=D_{1}^{+} f_{n}^{x}$, proving the claim. It follows that

$$
\phi\left(\left[g_{n}\right]-\left[f_{n}\right]\right)=\phi\left(\gamma_{n}\right),
$$

proving (24).

REMARK 5.15. Close inspection reveals that the image of $f_{n}$ is the set of all vertices of $G_{n}$ of the form $a_{i_{1}, \ldots, i_{n-1}}$, where we have added subscripts to the vertex $a \in G_{1}$. This set induces a subgraph of $G_{n}$ isomorphic to the graph $U_{n}$, where $U_{1}$ consists of a single vertex and $U_{n+1}=S_{n+3} U_{n}$ for all $n \geqslant 1$. Proposition 4.4 implies that $U_{n}$ is contractible for all $n$. Hence $\left[f_{n}\right]=0$ in $A_{n}\left(G_{n}\right)$, so in fact $\psi\left(\left[g_{n}\right]\right)=\overline{\phi\left(\gamma_{n}\right)}$.

Proof of Theorem 5.10. Let $G_{1}$ be any graph containing no 3- or 4-cycles. If $G_{1}$ contains no cycles, then $G_{n}$ is contractible for all $n$ by Example 2.11 and Proposition 4.4, so $\mathcal{H}_{n}\left(G_{n}\right)$ is trivial for all $n \geqslant 1$ by [4, Lemma 4.2], and the theorem is immediate. 
Suppose that $G_{1}$ contains a cycle. If $k<n$, then $\mathcal{H}_{k}\left(G_{n}\right)=0$ by Proposition 5.12(i), and the theorem is immediate. We prove the case $k=n$. Let $\left\{Z_{1,1}, \ldots, Z_{1, \ell}\right\}$ be the set of cycles of $G_{1}$, considered as subgraphs of $G_{1}$. For $i=1, \ldots, \ell$ and $n \geqslant 1$, let $Z_{n+1, i}=S_{n+3} Z_{n, i}$, considered as a subgraph of $G_{n+1}$. Lemma 5.13 can be easily generalized to obtain grid graphs $J_{n, i}$ and graph maps $\gamma_{n, i}: J_{n, i} \rightarrow Z_{n, i}$ satisfying the following for all $i$ and $n \geqslant 1$ :

(i) $\mathcal{H}_{n}\left(Z_{n, i}\right)=\left\langle\overline{\phi\left(\gamma_{n, i}\right)}\right\rangle$

(ii) $\Delta\left(\overline{\phi\left(\gamma_{n+1, i}\right)}\right)=(-1)^{n} \overline{\phi\left(\gamma_{n, i}\right)}$, where $\Delta: \mathcal{H}_{n+1}\left(G_{n+1}\right) \rightarrow \mathcal{H}_{n}\left(G_{n}\right)$ is the isomorphism from Proposition 5.12(ii).

Let

$$
\Upsilon_{n}=\left\{\overline{\phi\left(\gamma_{n, i}\right)}: i=1, \ldots, \ell\right\} .
$$

Example 5.3 and item (i) together say that $\mathcal{H}_{1}\left(G_{1}\right)$ is generated by $\Upsilon_{1}$. Item (ii) then implies that $\mathcal{H}_{n}\left(G_{n}\right)$ is generated by $\Upsilon_{n}$ for all $n \geqslant 1$. Lemma 5.13 can be easily generalized to show that the image of $\psi: A_{n}\left(G_{n}\right) \rightarrow \mathcal{H}_{n}\left(G_{n}\right)$ contains $\Upsilon_{n}$. Hence $\psi$ is surjective.

\section{Final REMARKS}

Our work leaves open several important questions. Let $G_{1}$ be a cycle graph of length 5 , and define the graphs $G_{n}$ as in (14). Our most immediate goal is to show that the map $\psi: A_{n}\left(G_{n}\right) \rightarrow \mathcal{H}_{n}\left(G_{n}\right)$ is an isomorphism for all $n$. Theorem 5.10 brings us halfway there; we leave the remaining half as a conjecture.

Conjecture 6.1. The map $\psi: A_{n}\left(G_{n}\right) \rightarrow \mathcal{H}_{n}\left(G_{n}\right)$ is injective for all $n$.

The main analogy guiding our intuition is to think of the graphs $G_{n}$ as playing the role of the $n$-sphere $S^{n}$ in classical topology. An obvious comparison to make is that $S^{n+1} \approx S S^{n}$, where $S$ is the usual suspension functor, since this mirrors the construction of $G_{n}$ via the discrete suspension functor $S_{t}$. Our analogy is strengthened by the fact that

$$
\mathcal{H}_{i}\left(G_{n}\right) \cong H_{i}\left(S^{n}\right)
$$

for all $i \leqslant n$. An important property of $S^{n}$, however, is $(n-1)$-connectedness. This is what allows one to apply the Hurewicz theorem and conclude that $\pi_{n}\left(S^{n}\right) \cong H_{n}\left(S^{n}\right)$. We suspect that $G_{n}$ is $(n-1)$-connected in a discrete sense.

Conjecture 6.2. If $i<n$, then $A_{i}\left(G_{n}\right)$ is trivial.

The usual way to prove that $S^{n}$ is $(n-1)$-connected is to invoke the cellular approximation theorem. A discrete analog of cellular approximation was proposed in [1]. An alternative proof of $(n-1)$-connectedness, such as the one in [13, Theorem 6.4.4], uses the homotopy excision theorem of Blakers and Massey.

TheOREM 6.3 (Blakers-Massey [10]). Suppose that a topological space $X$ is the union of open subspaces $A$ and $B$ with nonempty intersection $C=A \cap B$. If $(A, C)$ is $m$-connected and $(B, C)$ is n-connected, then the map

$$
\pi_{k}(B, C) \rightarrow \pi_{k}(X, A),
$$

induced by the inclusion $(B, C) \rightarrow(X, A)$, is surjective if $k \leqslant m+n$ and bijective if $k<m+n$.

This result is a powerful means of computing higher classical homotopy groups. In particular, it is a key ingredient in the proof of the Hurewicz theorem. We therefore expect some discrete version of Theorem 6.3 to appear in a proof of Conjecture 5.2, should it hold. At the very least, a discrete homotopy excision theorem would enable us to perform more elegant computations than are currently possible. 


\section{QUESTION 6.4. Is there a discrete homotopy excision theorem?}

A famous corollary of homotopy excision is the Freudenthal suspension theorem, which states that if $X$ is $n$-connected, then the map

$$
\pi_{k}(X) \rightarrow \pi_{k+1}(\Sigma X)
$$

induced by suspension, is an isomorphism for $k<2 n+1$ and surjective if $k=2 n+1$. This gives, for example, a sequence of maps

$$
\pi_{1}\left(S^{1}\right) \rightarrow \pi_{2}\left(S^{2}\right) \rightarrow \pi_{3}\left(S^{3}\right) \rightarrow \cdots
$$

in which the first map is surjective and all subsequent maps are isomorphisms. Ideally, a discrete homotopy excision theorem would give such a sequence for the graphs $G_{n}$, i.e. a sequence of maps

$$
A_{1}\left(G_{1}\right) \rightarrow A_{2}\left(G_{2}\right) \rightarrow A_{3}\left(G_{3}\right) \rightarrow \cdots
$$

in which the first map is surjective and the rest are isomorphisms. Since $A_{1}\left(G_{1}\right) \cong \mathbb{Z}$ and $A_{2}\left(G_{2}\right)$ is infinite by Corollary 5.11 , the first map must be an isomorphism as well. Thus it would follow that $A_{n}\left(G_{n}\right) \cong \mathbb{Z}$ for all $n$, confirming Conjecture 6.1 and further reinforcing the analogy between $G_{n}$ and $S^{n}$.

Acknowledgements. The author thanks Hélène Barcelo and Curtis Greene for extensive discussions and suggestions. The author also thanks Trevor Hyde for helpful comments on a draft of the paper.

\section{REFERENCES}

[1] Eric Babson, Hélène Barcelo, Mark de Longueville, and Reinhard Laubenbacher, Homotopy theory of graphs, J. Algebraic Combin. 24 (2006), no. 1, 31-44.

[2] Mathieu Baillif and Alexandre Gabard, Manifolds: Hausdorffness versus homogeneity, Proc. Amer. Math. Soc. 136 (2008), no. 3, 1105-1111.

[3] Hélène Barcelo, Valerio Capraro, and Jacob A. White, Discrete homology theory for metric spaces, Bull. Lond. Math. Soc. 46 (2014), no. 5, 889-905.

[4] Hélène Barcelo, Curtis Greene, Abdul Salam Jarrah, and Volkmar Welker, Discrete cubical and path homologies of graphs, Algebr. Comb. 2 (2019), no. 3, 417-437.

[5] - On the vanishing of discrete singular cubical homology for graphs, https://arxiv.org/ abs/1909.02901, 2019.

[6] Hélène Barcelo, Xenia Kramer, Reinhard Laubenbacher, and Christopher Weaver, Foundations of a connectivity theory for simplicial complexes, Adv. in Appl. Math. 26 (2001), no. 2, 97-128.

[7] Hélène Barcelo and Reinhard Laubenbacher, Perspectives on A-homotopy theory and its applications, Discrete Math. 298 (2005), no. 1-3, 39-61.

[8] Hélène Barcelo, Christopher Severs, and Jacob A. White, k-parabolic subspace arrangements, Trans. Amer. Math. Soc. 363 (2011), no. 11, 6063-6083.

[9] Hélène Barcelo and Shelly Smith, The discrete fundamental group of the order complex of $B_{n}$, J. Algebraic Combin. 27 (2008), no. 4, 399-421.

[10] Albert L. Blakers and William S. Massey, The homotopy groups of a triad. II, Ann. of Math. (2) $\mathbf{5 5}$ (1952), 192-201.

[11] Allen Hatcher, Algebraic topology, Cambridge University Press, Cambridge, 2002.

[12] Witold Hurewicz, Homotopie und Homologiegruppen, Proc. Akad. Wetensch. Amsterdam 38 (1935), 521-528.

[13] Tammo tom Dieck, Algebraic topology, EMS Textbooks in Mathematics, European Mathematical Society (EMS), Zürich, 2008.

Boв Lutz, Life Cycle Engineering, Inc., 4900 S. Broad St., Philadelphia, PA 19112, USA

E-mail : rlutz@lce.com 